\title{
Fbxo45-mediated degradation of the tumor-suppressor Par-4 regulates cancer cell survival
}

\author{
X Chen ${ }^{1}$, AA Sahasrabuddhe ${ }^{1}$, P Szankasi ${ }^{2}$, F Chung ${ }^{1}$, V Basrur ${ }^{1}$, VM Rangnekar ${ }^{3}$, M Pagano ${ }^{4,5}$, MS Lim $^{1,6}$ \\ and KSJ Elenitoba-Johnson ${ }^{\star, 1,6,7}$
}

Prostate apoptosis response protein 4 (Par-4) also known as PRKC apoptosis WT1 regulator is a tumor suppressor that selectively induces apoptosis in cancer cells. However, its post-translational regulation by ubiquitin-mediated proteolysis and the cellular machinery that is responsible for its proteasomal degradation are unknown. Using immunopurification and an unbiased mass spectrometry-based approach, we show that Par-4 interacts with the SPRY-domain containing E3 ubiquitin ligase Fbxo45 through a short consensus sequence motif. Fbxo45 interacts with Par-4 in the cytoplasm and mediates its ubiquitylation and proteasomal degradation. Fbxo45 silencing results in stabilization of Par-4 with increased apoptosis. Importantly, a Par-4 mutant that is unable to bind Fbxo45 is stabilized and further enhances staurosporine-induced apoptosis. Co-expression of Fbxo45 with Par-4 protects cancer cells against Par-4-induced apoptosis. Our studies reveal that Fbxo45 is the substratereceptor subunit of a functional E3 ligase for Par-4 that has a critical role in cancer cell survival.

Cell Death and Differentiation (2014) 21, 1535-1545; doi:10.1038/cdd.2014.92; published online 4 July 2014

F-box proteins are the substrate-specific adaptor subunits of SCF (Skp1, CUL1, F-box protein) E3 ubiquitin ligase complexes that promote proteasomal degradation of critical cellular regulators. ${ }^{1-6}$ Previous studies have shown that in contrast to other F-box proteins that assemble SCF ubiquitin ligase complexes, Fbxo45 forms an atypical ubiquitin ligase complex that contains Skp1 and the Ring-finger protein PAM (protein-associated with myc) also known as MYCBP2 (mycbinding protein 2). ${ }^{7,8}$ Fbxo45 has been linked to the proteasomal degradation of a few targets including $p 73^{9}$ and Munc13-1. ${ }^{10} \mathrm{FbxO} 45$ is one of only six F-box proteins that are conserved in metazoans and the only F-box protein known to contain a SPRY domain, ${ }^{3}$ which was first identified as a sequence repeat in the dual-specificity kinase splA and ryanodine receptors. ${ }^{11}$

SPRY domains are present in more than 150 human proteins that cover a wide spectrum of biological functions, including regulation of cytokine signaling and innate retroviral restriction. ${ }^{12}$ The crystal structure of the SPRY domain has been determined in complex with a 20 amino-acid residue peptide derived from the VASA protein. These observations led to the identification of a short sequence motif in Par-4 (ELNNNL) recognized by the SPRY domain. ${ }^{13}$

Par-4 was first identified in prostate cancer cells ${ }^{14}$ where it selectively induces apoptosis in androgen-independent and Ras-transformed cells but not in androgen-dependent cancer cells or normal prostate epithelial cells. ${ }^{15}$ The human Par-4 gene maps to chromosome 12q21, a region frequently deleted in malignancies, and encodes a protein $(38 \mathrm{kDa})$ containing conserved functional domains, which include two putative nuclear localization sequences (NLSs), designated NLS1 and NLS2; a leucine zipper domain spanning amino acids 290-332 in the C-terminal region and a nuclear export sequence in the $\mathrm{C}$-terminus. ${ }^{16}$

Par-4 localizes both to the cytoplasm and the nucleus in many cancer cells. ${ }^{15,16}$ Par-4 induces apoptosis in hormoneindependent cancer cells by enabling the translocation of Fas and Fas ligand (Fas/FasL) to the plasma membrane. ${ }^{17}$ In parallel, Par-4 translocates to the nucleus and inhibits NF- $\kappa$ Bmediated cell survival mechanisms. ${ }^{18}$ In other studies, Par-4 has been shown to function in the cytoplasm, where it represses NF- $\kappa$ B-dependent gene transcription by inhibiting the TNF $\alpha$-induced nuclear translocation of the p65 (Rel A) subunit by blocking the atypical protein kinase $C .^{19}$ The apoptotic function of Par-4 requires its phosphorylation at the $\mathrm{T} 155$ residue by PKA and nuclear translocation. ${ }^{20}$ On the other hand, phosphorylation of Par-4 by AKT inhibits apoptosis by interfering with the nuclear translocation of Par-4. ${ }^{21}$

Par-4 is a bona fide tumor suppressor, which exhibits a potent pro-apoptotic function in cancer cells. ${ }^{14,19}$ Accordingly, Par-4 is mutated or silenced in a variety of human cancers. ${ }^{22,23}$ Consistent with its pro-apoptotic effects, transgenic mice ubiquitously expressing the SAC domain of Par-4

\footnotetext{
${ }^{1}$ Department of Pathology, University of Michigan Medical School, 2037 BSRB 109 Zina Pitcher Place, Ann Arbor, Ml 48109, USA; ${ }^{2}$ ARUP Laboratories, 500 Chipeta Way, Salt Lake City, UT 84108, USA; ${ }^{3}$ Department of Microbiology, Immunology and Molecular Genetics, University of Kentucky, Lexington, KY 40508, USA; ${ }^{4}$ Department of Pathology, NYU Cancer Institute, New York University School of Medicine, 522 First Avenue, SRB1107, New York, NY 10016, USA; ${ }^{5}$ Howard Hughes Medical Institute, Chevy Chase, MD 20815, USA; ${ }^{6}$ Protein Folding Disease Initiative, University of Michigan Medical School, 109 Zina Pitcher Place, Ann Arbor, MI 48109, USA and ${ }^{7}$ Center for Computational Medicine and Bioinformatics, University of Michigan Medical School, 109 Zina Pitcher Place, Ann Arbor, Ml 48109, USA ${ }^{*}$ Corresponding author: KSJ Elenitoba-Johnson, Department of Pathology, University of Michigan Medical School, 2037 BSRB 109 Zina Pitcher Place, Ann Arbor, MI 48109, USA. Tel: +734 615-4388; Fax: +734 615-9666; E-mail: kojoelen@ umich.edu

Abbreviations: Par-4, Prostate apoptosis response protein 4; PAWR, PRKC apoptosis WT1 regulator; PAM, protein-associated with myc; SCF, Skp1, CUL1, F-box protein; MYCBP2, myc-binding protein 2; NLS, nuclear localization sequence; LC-MS/MS, liquid chromatography-tandem mass spectrometry; mES, mouse embryonic stem; SOCS, suppressor of cytokine signaling; WCE, whole-cell extract

Received 13.1.14; revised 09.5.14; accepted 23.5.14; Edited by A Peschiaroli; published online 04.7.14
} 
are resistant to the growth of spontaneous and inducible tumors. ${ }^{24,25}$ Emerging data have implicated Par-4-induced multinucleation as a mechanism of cell death in oncogeneaddicted cells and establish Par-4 as a negative regulator of breast cancer recurrence. ${ }^{26}$

In this study, we investigated whether endogenous Par-4 in cancer cells is regulated by proteasomal degradation and identified the cellular mechanism that regulates its ubiquitinmediated proteolysis thereby controlling its apoptotic function.

\section{Results}

Fbx045 specifically interacts with Par-4. F-box proteins are the substrate-specific adaptor subunits of SCF E3 ubiquitin ligase complexes. ${ }^{1-6}$ To identify the candidate substrates of the Fbxo45 ubiquitin ligase, we isolated Fbxo45 immunocomplexes by transient expression of HA-tagged Fbxo45 in human U87MG cell, followed by liquid chromatography-tandem mass spectrometry (LC-MS/MS). The LC-MS/MS data revealed known members of the atypical SCF complex, Fbxo45, Skp1 and MYCBP2. The LC-MS/MS analysis also identified peptides corresponding to Par-4 (PAWR; Figure 1a). These peptides were not found in the negative control (HA-tag only) immunopurification suggesting Par-4 as an interactor of Fbxo45.

We further examined the binding of Fbxo45 and Par-4 by immunoprecipitation and western blot analysis. We cotransfected V5-tagged Par-4 and HA-tagged Fbxo45 into 293T cells and demonstrated reciprocal co-immunoprecipitation of both proteins (Figure 1b). To examine whether Par-4 interacts with endogenous Fbxo45, we expressed Flagtagged Par-4 in 293T cells and performed immunoprecipitation and western blot analysis. The results indicate that Par-4 interacts with endogenous Fbxo45 (Figure 1c).

To assess the ability of Fbxo45 to reciprocally interact with endogenous Par-4 and investigate the specificity of the interaction, we screened eight human F-box proteins and found that only Fbxo45 was able to co-immunoprecipitate endogenous Par-4. (Figure 1d, Supplementary Figures 1a and b).

Consistent with previous findings showing that Fbxo45 forms a complex with Skp1 and PAM (and its orthologs) in Drosophila, $^{27}$ Caenorhabditis elegans $^{7}$ and Mus musculus. ${ }^{8}$ We confirmed that human Fbxo45 interacts with endogenous PAM and Skp1, but not with Cullin1 or Rbx1, by immunoprecipitation and western blotting (Supplementary Figure 1d). Yeast two-hybrid analyses revealed that Fbxo45 bound to Skp1 through the F-box domain, and PAM via the SPRY domain (Supplementary Figure 1e). Overall, these experiments confirm that human Fbxo45 forms an atypical multicomponent E3 ligase with Skp1 and PAM in a complex similar to what has been reported in other species.

\footnotetext{
Fbxo45 interacts with Par-4 through SPRY domain in the cytoplasm. Fbxo45 is the only F-box protein known to contain a SPRY domain. To determine whether the SPRY domain mediates the interaction of Fbxo45 with Par-4, we generated a series of truncated Fbxo45 proteins and performed binding experiments. The results demonstrated that the SPRY domain of Fbxo45 specifically mediates the interaction between Par-4 and Fbxo45 (Figure 2a).
}

Given that Par-4 localizes both to the cytoplasm and the nucleus in many cancer cells and Par-4-mediated apoptosis requires the nuclear function of Par-4 protein, ${ }^{15}$ we sought to determine the intracellular localization of its interaction with Fbxo45. To this end, the interaction between Par-4 and Fbxo45 was studied by immunoprecipitation and western blot analysis using lysates from cytoplasmic and nuclear fractions. The interaction was only found in the cytoplasm (Figure $2 b$ ). Phosphorylation of Par-4 by AKT has been shown to interfere with its nuclear translocation by promoting Par-4 binding to protein 14-3-3 and restricting its localization to the cytoplasm. ${ }^{21}$ Accordingly, we treated PC-3 cells with AKT kinase inhibitor (CAL-101) to promote Par-4 translocation to the nucleus. As shown in Figure 2c, CAL-101 treatment promoted Par-4 nuclear translocation and diminished the interaction between Par-4 and Fbxo45 in the cytoplasm.

Fbxo45 regulates the stability of Par-4 in the cytoplasm through proteasomal degradation. Having observed the interaction with the E3 ligase Fbxo45, we sought to determine whether Par-4 was regulated by proteasomal degradation. To examine this possibility, HeLa cells were treated with the proteasomal inhibitor MG132. After $1 \mathrm{~h}$ treatment, cells were exposed to cycloheximide for different times. Whole-cell extracts (WCEs) were analyzed to investigate the half-life of Par-4. Our results indicated that Par-4 has a half-life of $\sim 2 \mathrm{~h}$ in HeLa cells; after the treatment with MG132, Par-4 was stabilized and did not undergo significant degradation even at $6 \mathrm{~h}$ (Figure 3a). These results suggest that Par-4 levels are regulated by proteasome-mediated degradation.

Having observed a specific interaction between Fbxo45 and Par-4 (Figure 1), we investigated whether Fbxo45 regulated the stability of Par-4. HeLa cells were transiently transfected with Scrambled or siRNA smart pool targeting Fbxo45. After $48 \mathrm{~h}$ of transfection, Fbxo45-depleted cells and control cells were treated with cycloheximide for different times. WCEs were analyzed to investigate the half-life of Par-4. As seen in Figure $3 b$, Par-4 has a half-life of $\sim 3 \mathrm{~h}$ in the control cells; but in the Fbxo45 knockdown cells, Par-4 was stabilized and its half-life was significantly increased $(>6 \mathrm{~h} ; P<0.05)$. To confirm this observation, we stably silenced Fbxo45 in mouse embryonic stem (mES) cells using Fbxo45 shRNA and evaluated the half-life of Par-4. As shown in Figure 3c, mES cells expressing Fbxo45 shRNA showed Par-4 stabilization, whereas those expressing control shRNA showed faster Par-4 turnover with $>80 \%$ decrease in Par-4 levels at 4-6 h of cycloheximide treatment.

In corollary experiments, we demonstrated that ectopic Fbxo45 expression promoted the degradation of Par-4 in Hela cells (Supplementary Figure 2a). Interestingly, silencing or ectopic expression of another SPRY-contained protein TRIM 21 did not regulate the stabilization of Par-4 (Supplementary Figure 2b). In addition, endogenous Par-4 was not stabilized by the neddylation inhibitor (MLN4924) treatment (Supplementary Figure 4), thus suggesting that Par-4 proteasomal degradation is not mediated by a Cullin-ring ligase.

To determine whether the regulation between Fbxo45 and Par-4 also occurred in the cytoplasm, we assessed the level of Par-4 after treatment with the AKT inhibitor CAL-101. As seen 
a

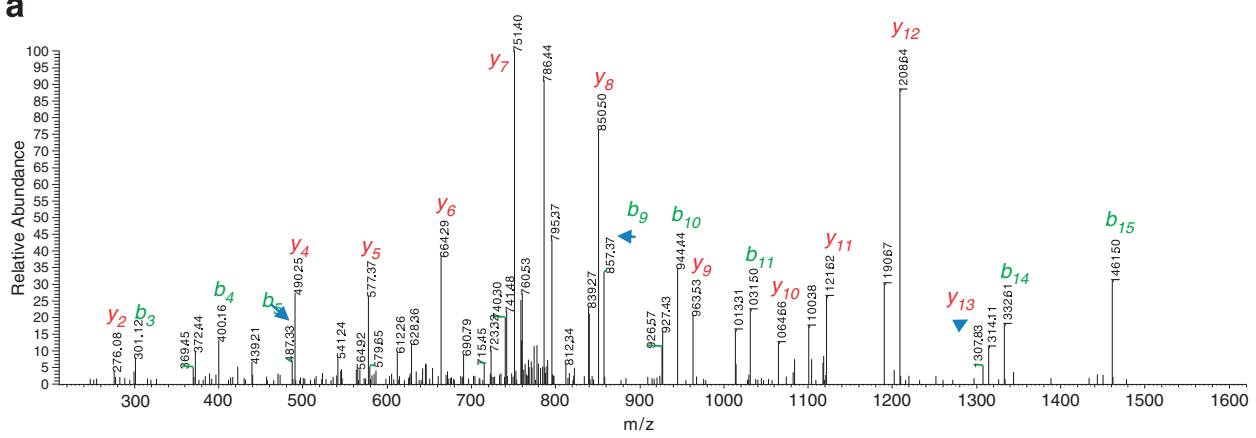

>spIQ96IZOIPAWR_HUMAN PRKC apoptosis WT1 regulator protein OS=Homo sapiens

$\mathrm{GN}=\mathrm{PAWR} P E=1 \mathrm{SV}=1$

MATGGYRTSSGLGGSTTDFLEEWKAKREKMRAKQNPPGPAPPGGGSSDAAGKPPAGALGTPAAAAANELNNNL

PGGAPAAPAVPGPGGVNCAVGSAMLTRAAPGPRRSEDEPPAASASAAPPPQRDEEEPDGVPEKGKSSGPSARK

GKGQIEKRKLREKRRSTGVVNIPAAECLDEYEDDEAGQKERKREDAITQQNTIQNEAVNLLDPGSSYLLQEPP

RTVSGRYKSTTSVSEEDVSSRYSRTDRSGFPRYNRDANVSGTLVSSSTLEKKIEDLEKEVVRERQENLRLVRL

MQDKEEMIGKLKEE IDLLNRDLDDIEDENEQLKQENKTLLKVVGQLTR

b

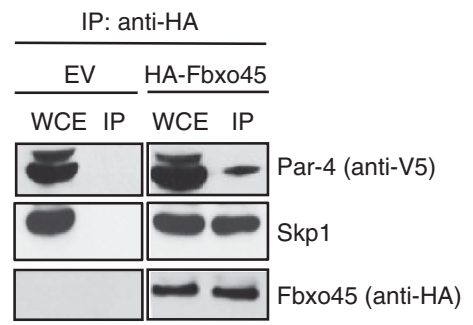

C

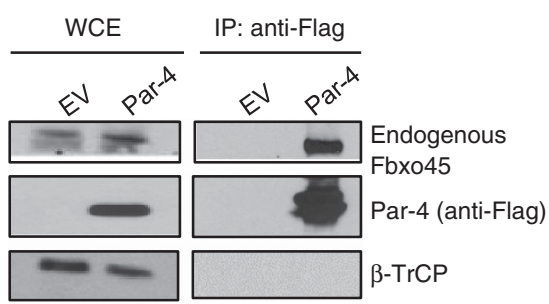

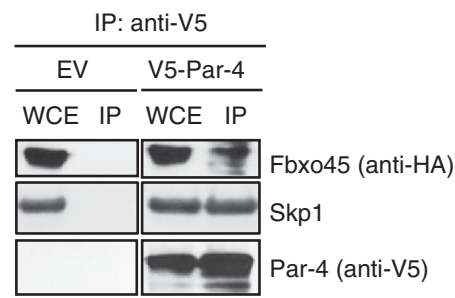

d

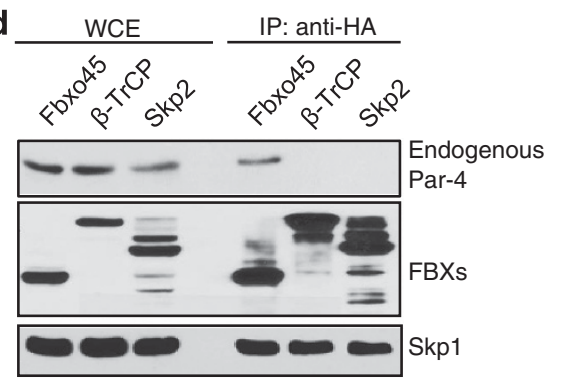

Figure 1 Par-4 interacts specifically with Fbx045. (a) Fbxo45 immunocomplex, isolated from U87MG cells expressing HA-Fbx045, was resolved on a SDS-PAGE. Following in-gel digestion with trypsin, LC-MS/MS data were acquired on a linear ion-trap instrument (LTQ Orbitrap, Thermo Fisher). A representative MS/MS spectrum belonging to PAWR (Par-4) ${ }^{255}$ DANVSGTLVSSSTLEK ${ }^{270}$ ), (precursor $[\mathrm{MH}]^{+2}=804.41 \mathrm{~m} / \mathrm{z}$ ) is shown (upper panel). Observed $b$ - and $y$-ions are indicated. Bottom panel shows the complete amino-acid sequence of PAWR (Par-4) and identified peptides (underlined). (b) Par-4 co-immunoprecipitates Fbx045. 293T cells were co-transfected with HA-tagged Fbx045 and V5-tagged Par-4. WCEs were subjected to immunoprecipitation (IP) with anti-V5 resin or anti-HA resin followed by western blot analysis with indicated antibodies. (c) Par-4 interacts with endogenous Fbx045. 293T cells were transfected with empty vector (EV) or the indicated Flag-tagged Par-4. WCEs were subjected to IP with anti-Flag resin followed by western blot analysis with indicated antibodies. (d) Par-4 specifically interacts with Fbxo45. 293T cells were transfected with EV or the indicated HA-tagged (Fbxo45, $\beta$-TrCP, Skp2) F-box protein constructs (FBPs). WCEs were subjected to IP with anti-HA resin followed by western blot analysis with indicated antibodies

in Figures 3d and e, inhibition of AKT kinase activity resulted in the accumulation of Par-4. Further, we found that the accumulation only occurred in the nucleus (Figure $3 e$ ). Altogether, these results strongly indicate that Fbxo45 interacts and promotes the degradation of Par-4 in the cytoplasm, and that translocation to the nucleus may protect Par-4 from degradation mediated by Fbxo45.

Fbxo45 binds to Par-4 through its VASA-like sequence. Substrate-recognition sites for the SPRY have been identified for human Par-4 (ELNNNL) and for the Drosophila orthologue GUSTAVUS binding to the DEAD-box RNA helicase VASA (DINNNN). ${ }^{28}$ To determine whether the interaction between Fbxo45 and Par-4 occurs via the VASA-like sequence (ELNNNL) of Par-4, we generated a Par-4 deletion mutant (Par-4 $\Delta 120$ ) and a point mutant (Par-4 3A), in which the critical E-L-N-N-N residues in the VASA-like sequence of Par-4 were mutated to $A-L-A-N-A$ residues. We then performed binding experiments with these constructs to map the Fbxo45 recognition motif in Par-4 (Figure $4 \mathrm{a}$ ). These experiments 
a

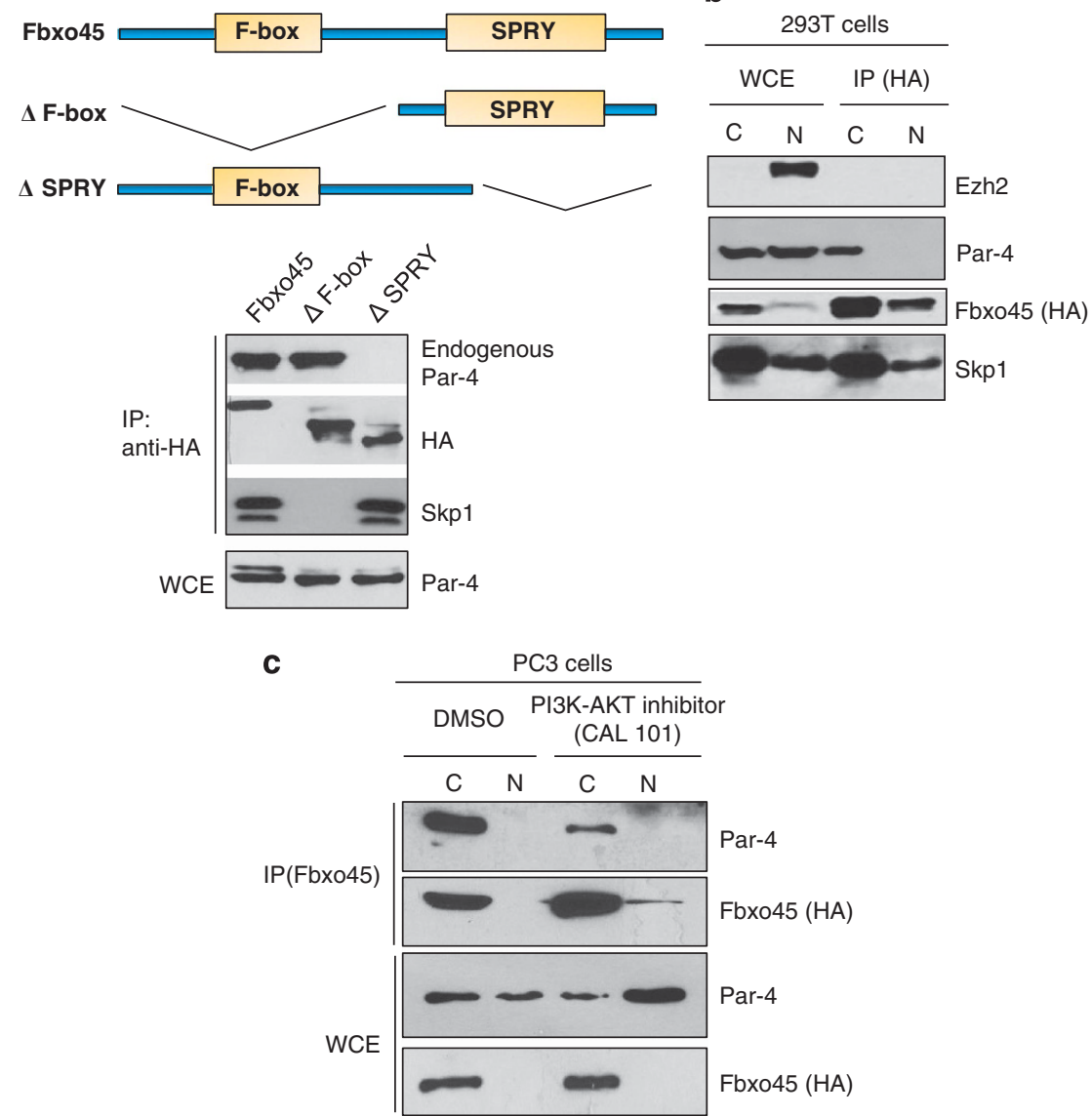

Figure 2 Par-4 interacts specifically with Fbxo45 in the cytoplasm through SPRY domain. (a) Par-4 interacts Fbxo45 via SPRY domain. 293T cells were transfected with HAtagged full-length Fbxo45 or truncated Fbxo45. WCEs were subjected to IP with anti-HA resin followed by western blot analysis with indicated antibodies. (b) Par-4 interacts with Fbxo45 in the cytoplasm. 293T cells were transfected with HA-tagged Fbx045. At $48 \mathrm{~h}$ after transfection, cell extracts from the cytoplasm or nuclei were subjected to IP with anti-HA resin followed by western blot analysis with indicated antibodies. (c) 293T cells were transfected with HA-tagged Fbxo45. At 48 h after transfection, cells were treated with AKT inhibitor (CAL-101; $10 \mu \mathrm{M}, 4 \mathrm{~h}$ ), cell extracts from the cytoplasm or nuclei were subjected to IP with anti-HA resin followed by western blot analysis with indicated antibodies

revealed that neither the VASA-like sequence deletion mutant (Par-4 $\Delta 120)$ nor the VASA-like sequence triple point mutant (Par-4 3A) were able to interact with Fbxo45 in reciprocal immunoprecipitation experiments (Figure 4b). To further confirm the requirement of the VASA-like sequence for the direct interaction between Fbxo45 and Par-4, we synthesized recombinant proteins and conducted in vitro binding experiments. As shown in Supplementary Figure 1c, recombinant Par-4 (WT), but not Par-4 (3A), directly interacts with Fbxo45 (Supplementary Figure 1c).

Moreover, we found that the Par-4 3A mutant, which does not bind Fbxo45, exhibited a longer half-life $(>6 \mathrm{~h})$ than the wild-type Par-4 in HeLa cells ( $\sim 3 \mathrm{~h}$; Figure $4 \mathrm{c})$. Finally, in vivo and in vitro ubiquitylation assays showed that immunopurified Fbxo45 promoted ubiquitylation of wild-type Par-4, but not the Par-4 3A mutant (Figure 4d, Supplementary Figure $3 b$ ). To confirm that Par-4 is ubiquitylated by Fbxo45, we stably silenced Fbxo45 in Hela cells using Fbxo45 shRNA, and assessed Par-4 ubiquitylation. As shown in Supplementary Figure $3 a$, silencing of Fbxo45 decrease the ubiqutylation of Par-4. Taken together, these data indicate that Fbxo45 mediates the ubiquitin-mediated degradation of Par-4 through its VASA-like sequence.
Fbxo45 regulates apoptosis in cancer cells by ubiquitin-mediated proteolysis of Par-4. To investigate whether Fbxo45-mediated degradation of Par-4 alters its apoptotic activity, we expressed the empty vector, the Par-4 (WT) and the Par-4 (3A) mutant in HeLa cells using an inducible lentivirus system. The cells expressing GFP-Par-4 or GFP-Par-4 (3A) were examined by western blot analysis (Figure 5a, right upper panel). Further, we tested the sensitivity of HeLa cells expressing the empty vector, the Par-4 (WT) and the Par-4 (3A) mutant to staurosporine-induced apoptosis by flow cytometry. Annexin V/7-AAD staining showed that ectopic expression of wildtype Par-4 enhanced staurosporine-induced apoptosis compared with that of empty vector. Expression of the Par-4 (3A) mutant resulted in a significant increase (twofold; $P<0.05$ ) in the number of apoptotic cells, suggesting that the Par-4 (3A) mutant may accumulate in the cells and promote staurosporine-induced apoptosis (Figure 5a, left panel and bar graph). Furthermore, TUNEL assays (Figure 5b) and western blot analysis for cleaved PARP-1 (Supplementary Figure 5) showed that the expression of the Par-4 $3 \mathrm{~A}$ mutant enhanced staurosporine-induced apoptosis by twofold $(P<0.05)$ compared with wild-type Par-4. In corollary 
a

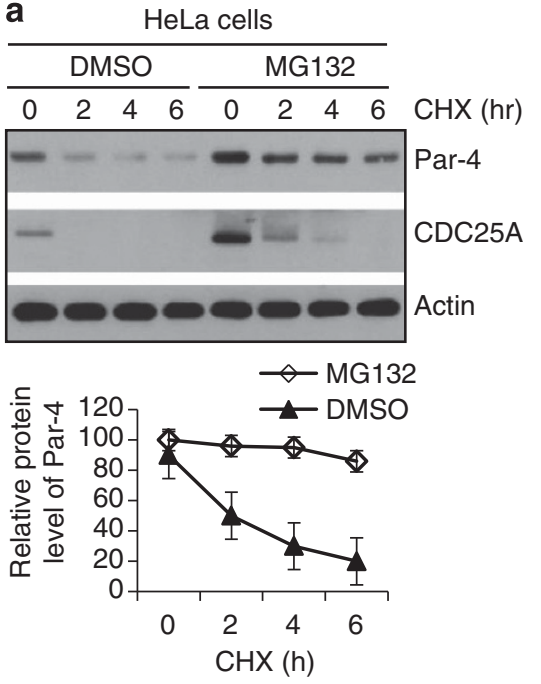

C
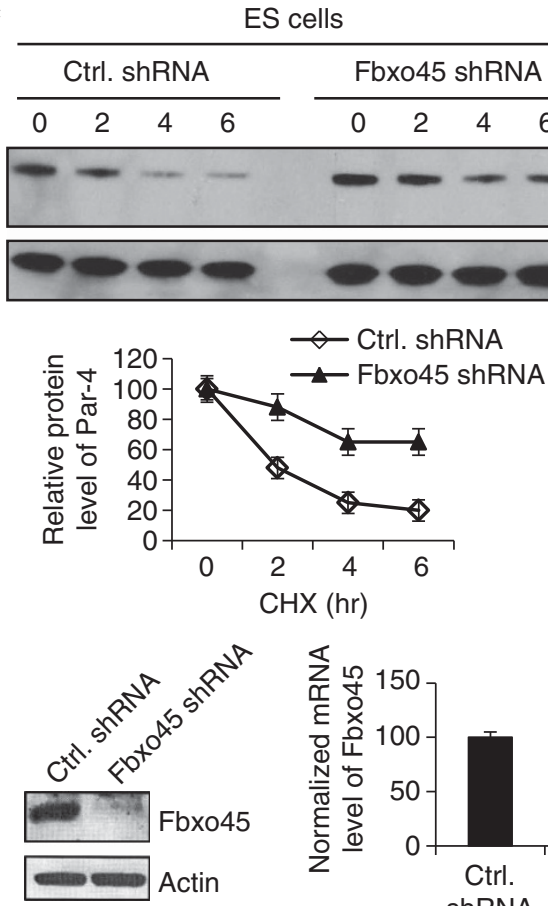

b
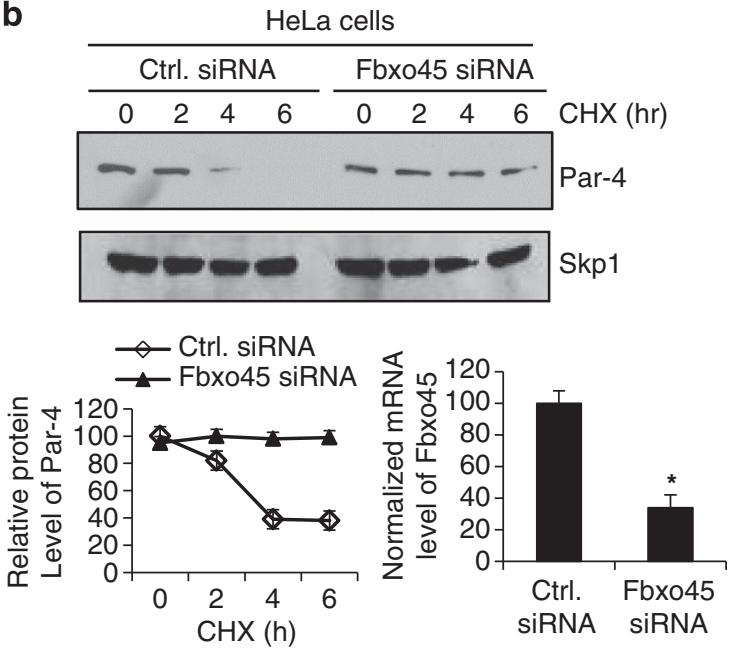

d

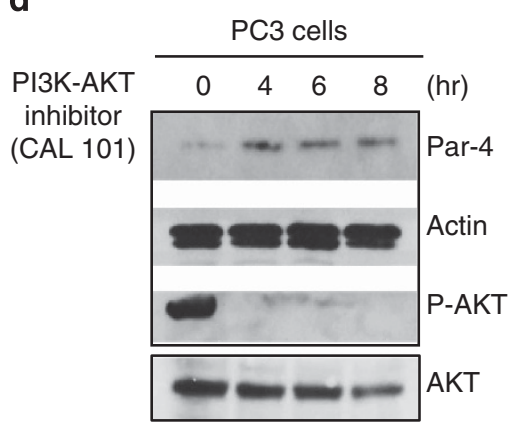

e

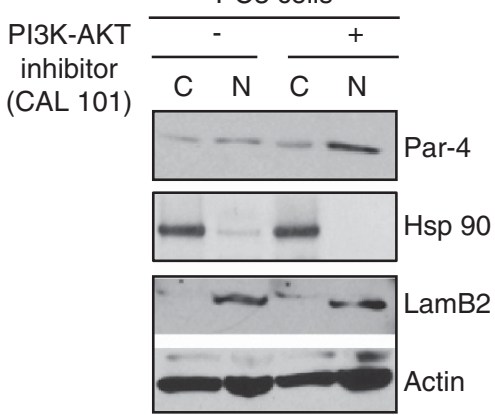

Figure 3 Fbxo45 regulates the stability of Par-4 by proteasomal degradation. (a) Par-4 is stabilized by MG132 treatment. Extract preparation and drug treatment were performed as previously described. ${ }^{34}$ HeLa cells were treated with the proteasome inhibitor MG132 or DMSO. After $1 \mathrm{~h}$ treatment, cells were further treated with cycloheximide (CHX) for different times. WCEs were analyzed by blotting with indicated antibodies to detect the levels of endogenous Par-4 and control proteins. CDC25A (a substrate of $\beta$-TrCP) was used as a positive control. The graph shows the level of endogenous Par-4. Error bars represent \pm S.D. $(n=3)$. ${ }^{*} P<0.05$. (b) Silencing of Fbxo45 stabilizes Par-4 in HeLa cells. Fbx045 knockdown cells (bar graph shows RNA level of Fbx045) and control cells were treated with CHX for different times. WCEs were analyzed by blotting with indicated antibodies to detect the levels of endogenous Par-4. The levels of Fbxo45 were determined by western blot analysis and RT-PCR. The graph shows the level of endogenous Par-4. Error bars represent \pm S.D. $(n=3)$. ${ }^{*} P<0.05$. (c) Silencing of Fbxo45 stabilizes Par-4 in mES cells. Fbxo45-depleted cells (the protein level and RNA level of Fbxo45 were detected by western blot analysis and RT-PCR) and control cells were treated with $\mathrm{CHX}$ for different times. WCEs were analyzed by western blot analysis with indicated antibodies to detect the steady-state level of endogenous Par-4. The levels of Fbxo45 were determined by western blot analysis and RT-PCR (right panel). The graph shows the half-life of Par-4. Error bars represent \pm S.D. $(n=3)$. ${ }^{*} P<0.05$. (d) Inhibition of phosphorylation by AKT stabilizes Par-4. PC3 cells were treated with AKT inhibitor $(\mathrm{CAL}-101 ; 10 \mu \mathrm{M})$ for different time periods. WCEs were analyzed by western blot analysis with indicated antibodies to detect the steady-state level of endogenous Par-4 and other control proteins. (e) Stabilization of Par-4 through AKT inhibition occurs in the nuclear fraction. PC3 cells were treated with AKT inhibitor (CAL-101; $10 \mu \mathrm{M})$ for $4 \mathrm{~h}$. Extracts from cytoplasmic or nuclear fraction were analyzed by blotting with indicated antibodies to detect the steady-state level of endogenous Par-4 and other proteins

experiments, we demonstrated that silencing of Fbxo45 significantly enhanced staurosporine-induced apoptosis compared with that of empty vector (Supplementary Figure 6).
To gain further insight into how Fbxo45-mediated degradation of Par-4 could regulate cancer cell apoptosis, we coexpressed Fbxo45 (WT) or Fbxo45 ( $\triangle \mathrm{SPRY}$ ) with Par-4 in HeLa cells. As expected, Annexin V/7-AAD staining showed 
that ectopic expression of wild-type Par-4 yielded higher apoptosis rates $(20.9 \%)$ in comparison to control cells $(6.6 \%)$, whereas co-expression of Fbxo45 (WT) and Par-4 reverted the apoptosis rate to near control levels (13.2\%; $P<0.05$; Figure $5 \mathrm{c}$ ). However, reversion of the apoptosis rate was not observed when Fbxo45 ( $\triangle$ SPRY) was co-expressed with Par-4 (Supplementary Figure 7).

Fbx045 inhibits Par-4-induced growth suppression. To investigate whether Fbxo45-mediated destruction of Par-4

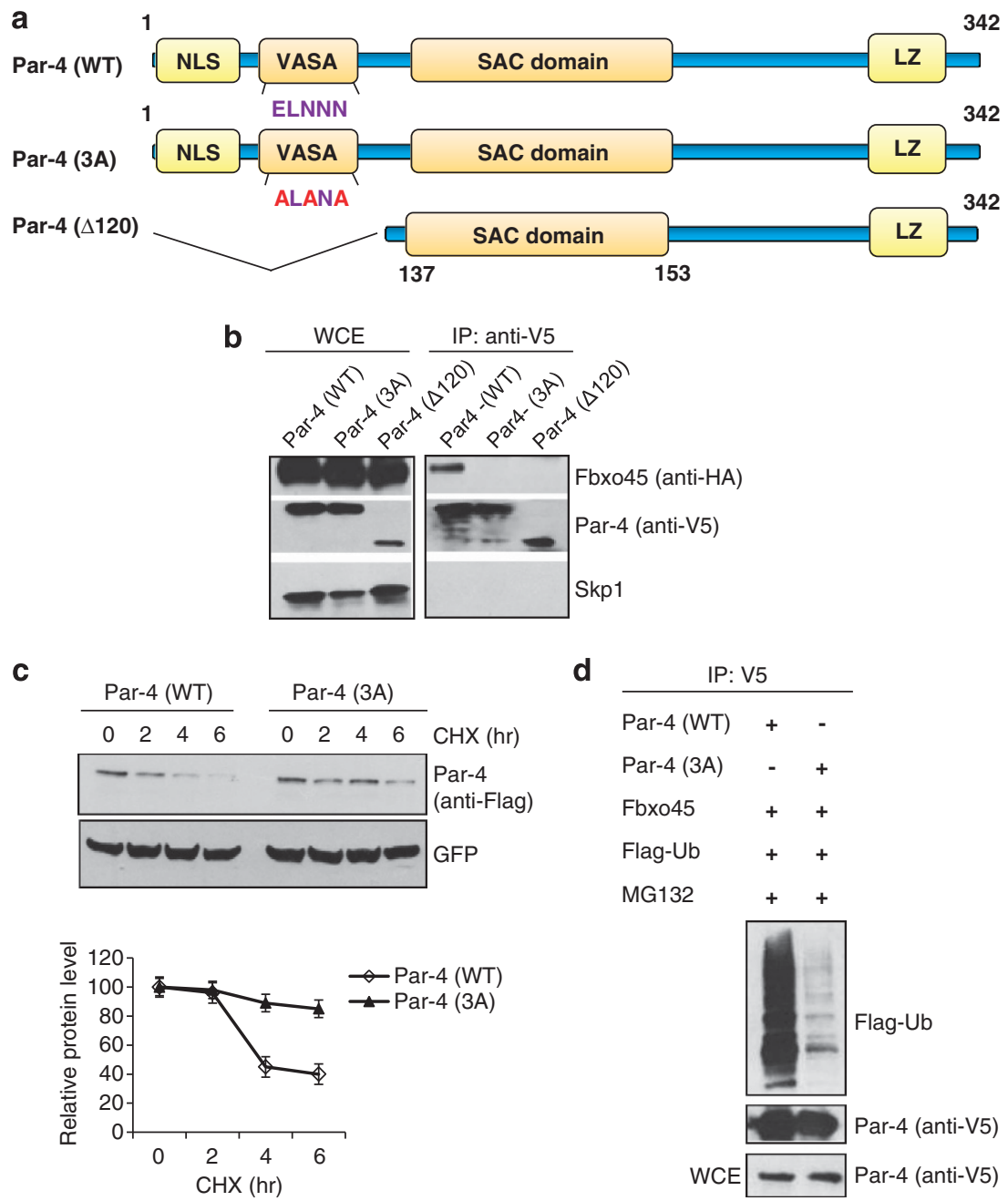

Figure 4 Fbxo45 regulates Par-4 degradation through a VASA-like sequence. (a) Schematic representation of various constructs used for identification of Fbxo45 interaction domain of Par-4. (b) Identification of Fbxo45-binding site. 293T cells were transfected with HA-tagged Fbxo45 and the indicated V5-tagged truncated Par-4. At 48 $\mathrm{h}$ after transfection, WCEs were subjected to IP with anti-V5 resin followed by blotting with indicated proteins. (c) Par-4 (3A) is not degraded by Fbxo45. HeLa cells were infected with Flag-GFP-tagged Par-4 or Flag-GFP-tagged Par-4 (3A) lentiviral inducible constructs to generate cell lines stably expressing wild-type or the mutant Par-4 proteins. After $24 \mathrm{~h}$ of induction with doxycycline, the cells were treated with $\mathrm{CHX}$ for different times. WCEs were analyzed by blotting with indicated antibodies to detect the steady-state level of exogenous Par-4 and control proteins. The graph shows the half-life of Par-4. Error bars represent \pm S.D. $(n=3)$. (d) Wild-type Par-4, but not mutant Par-4 (3A), is in vivo ubiquitylated by Fbx045. 293T cells were transfected with HA-tagged Fbx045, FLAG-tagged ubiquitin and V5-tagged wild-type Par-4 or mutant Par-4 (3A). At 48h after transfection, WCEs were subjected to IP with anti-V5 resin followed by blotting with the indicated antibodies

Figure 5 Fbxo45 regulates apoptosis in cancer cells by ubiquitin-mediated proteolysis of Par-4. (a) HeLa cells from Figure 4c were treated with doxycycline to induce exogenous protein expression. After $24 \mathrm{~h}$, either WCEs were analyzed by blotting with indicated antibodies (right upper panel) or the culture medium was replaced with fresh medium containing $1 \mu \mathrm{M}$ staurosporine or DMSO. Following $1.5 \mathrm{~h}$ of treatment, cells were collected for flow cytometric analysis after staining by APC-annexin V/7-AAD. B1, B2, B3 and B4 represent quadrants for dead, late apoptotic, viable, and early-apoptotic cells, respectively. Histogram shows apoptosis induction (\% total) in different groups. Data represent means S.D. of three independent experiments. Error bars represent \pm S.D. $(n=3)$. ${ }^{*} P<0.05$. (b) HeLa cells from Figure $4 \mathrm{c}$ were treated with doxycycline to induce exogenous protein expression. After $24 \mathrm{~h}$, the culture medium was replaced with fresh medium containing $1 \mu \mathrm{M}$ staurosporine or DMSO. Following $1.5 \mathrm{~h}$ of treatment, cells were collected for TUNEL staining (red) assay. Apoptotic cells were quantified and expressed as a percentage of the total number of cells. Data represent means S.D. of three independent experiments. Error bars represent \pm S.D. $(n=3) .{ }^{*} P<0.05$. (c) HeLa cells from Figure $4 \mathrm{c}$ were transfected with Fbxo 45 or the empty vector. After $24 \mathrm{~h}$, cells were treated with doxycycline to induce the exogenous protein expression. After $24 \mathrm{~h}$, the culture medium was replaced with fresh medium containing $1 \mu \mathrm{M}$ staurosporine or DMSO. Following $1.5 \mathrm{~h}$ of treatment, cells were collected for western blot analysis and flow cytometry using APC-annexin V/7-AAD staining. B1, B2, B3 and B4 represent quadrants for dead, late apoptotic, viable and early apoptotic cells, respectively. Histogram shows apoptosis induction (\% total) in different groups. Data represent means and S.D. of three independent experiments. Error bars represent \pm S.D. $(n=3)$. ${ }^{*} P<0.05$ 
a

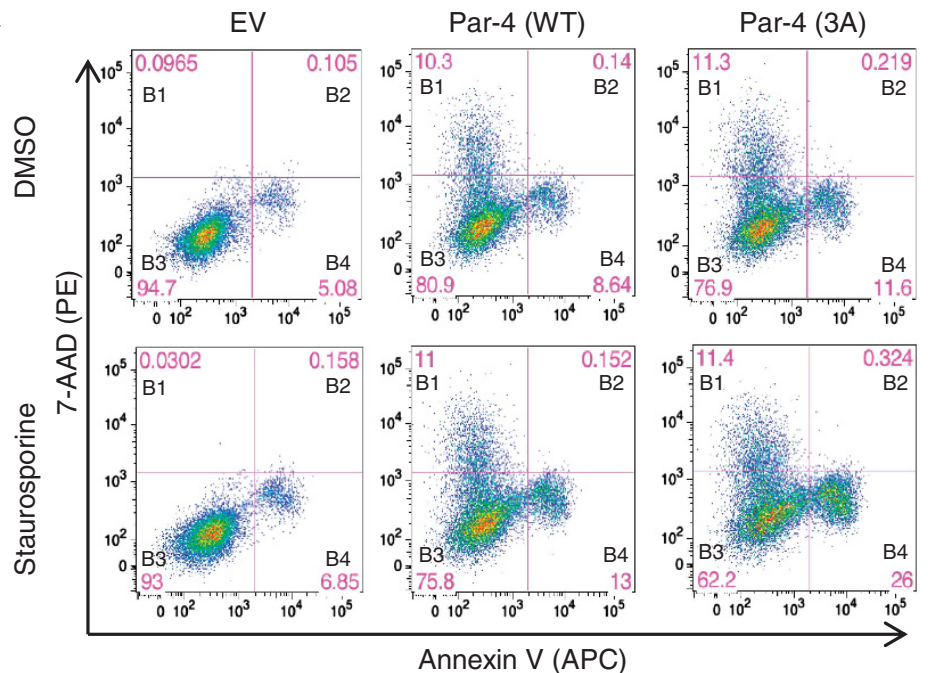

b
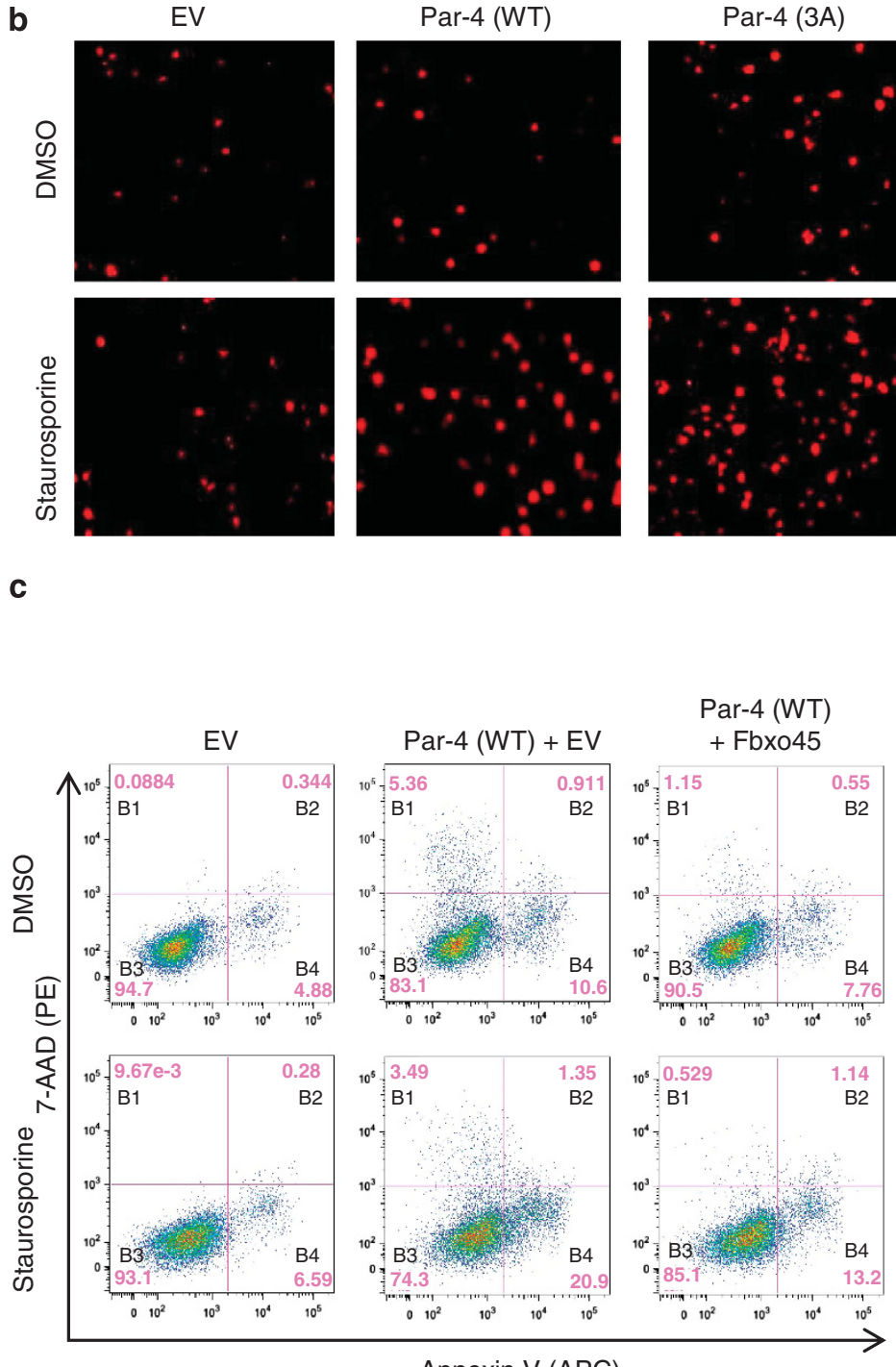

Par-4 (3A)
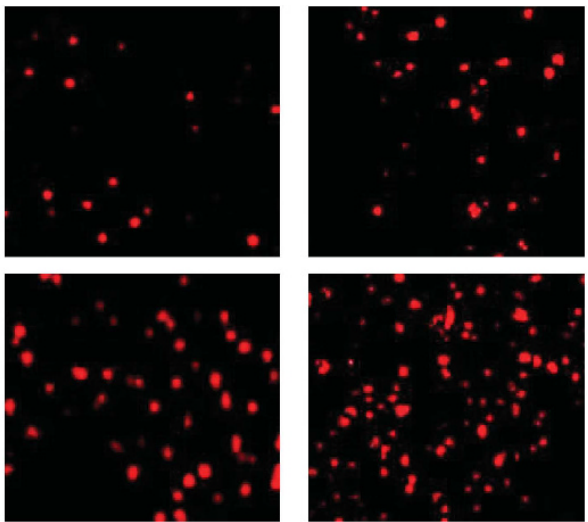
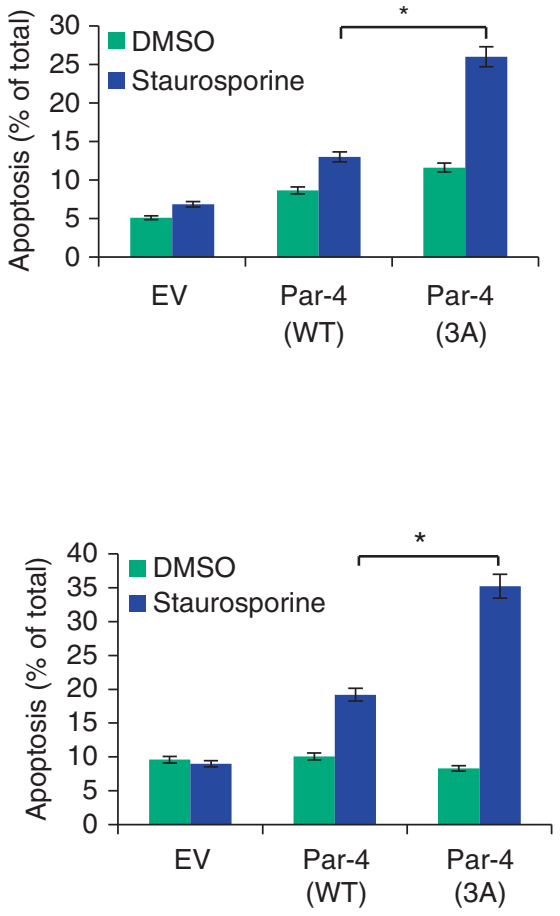

(3A)
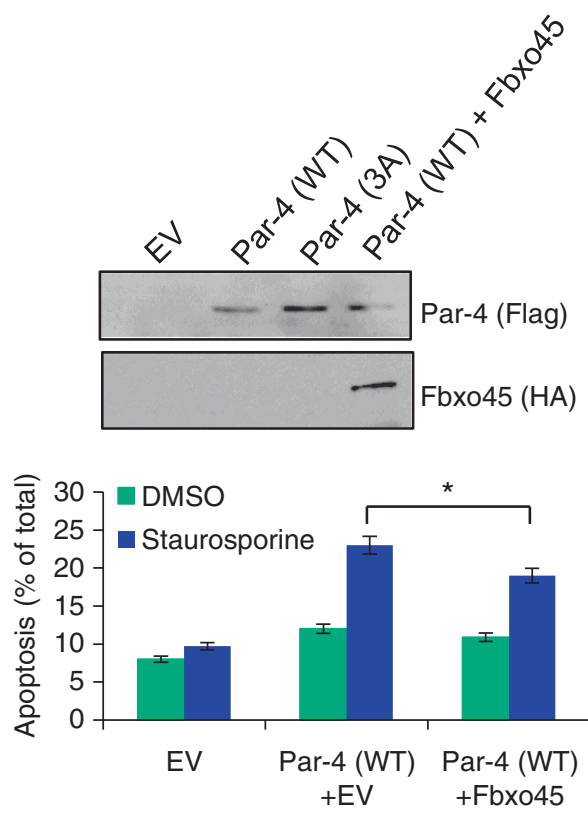
a

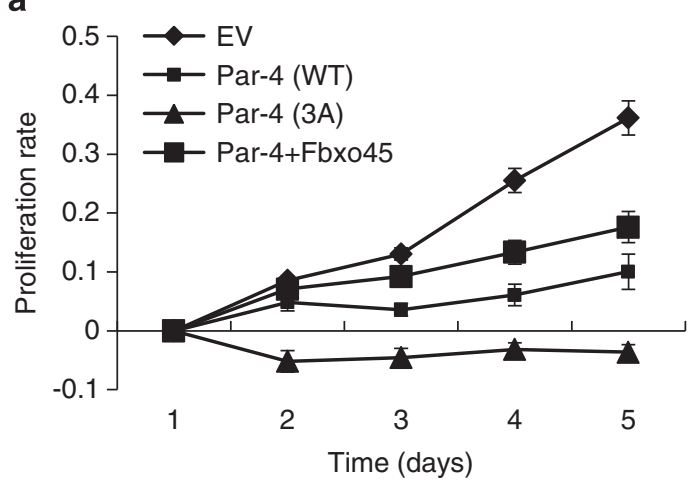

b

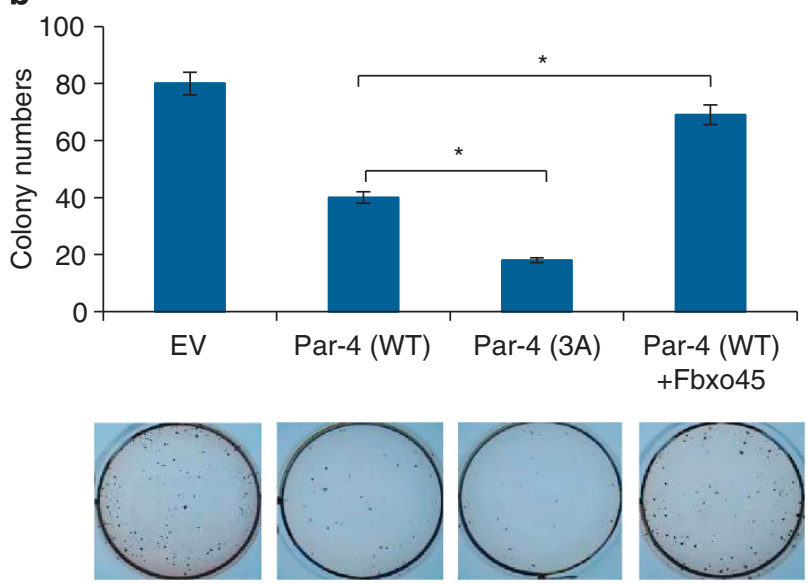

Figure 6 Fbxo45 inhibits Par-4-induced growth suppression. (a) HeLa cells from Figure 5 were seeded and analyzed for proliferation. The data shown are derived from four independent experiments $( \pm$ S.D., $P<0.01$, compared with cells expressing EV and Par-4 (WT)). (b) HeLa cells from Figure 5 were tested for anchorage-independent growth in a soft-agar colony assay. Viable colonies after 3 weeks were counted and the data $( \pm$ S.D.) from three independent experiments were presented $(P<0.01$, compared with cells expressing EV and Par-4 (WT))

inhibits its tumor-suppressor function, we used the cell lines from Figure 4c, which stably expressed either empty vector, the Par-4 (WT) or the Par-4 (3A) mutant, and a cell line wherein Fbxo45 was co-expressed with Par-4 (WT). Par-4 (3A)expressing cells showed a decreased rate of cell proliferation when compared with empty vector cells or Par-4 (WT)expressing cells. By contrast, cells co-expressing Fbxo45 and Par-4 (WT) showed an increased rate of cell proliferation compared with Par-4 (WT) only expressing cells (Figure 6a).

In addition, we analyzed the colony formation capacity of the cells from Figure $6 a$ by soft-agar colony formation assays. As shown in Figure 6b, Fbxo45 markedly increased the colony-forming capacity of HeLa cervical cancer cells suppressed by Par-4 overexpression. In corollary experiments, HeLa cells from Supplementary Figures 6 and 7 were tested for colony formation capacity. In this regard, silencing of Fbxo45 decreased the colony-forming capacity of HeLa cervical cancer cells (Supplementary Figure 8a), but Fbxo45 $(\triangle \mathrm{SPRY})$ did not increase the colony-forming capacity of HeLa cervical cancer cells suppressed by Par-4 overexpression (Supplementary Figure 8b).

\section{Discussion}

Post-translational modifications including ubiquitylation and phosphorylation regulate the function of key signaling molecules by modulating their activity, localization and protein stability. ${ }^{29}$ Par-4 is a tumor suppressor and a critical regulator of tumor cell survival. ${ }^{14,26}$ Subtle changes in regulation and alteration of Par-4 expression and localization may impact its biological function as a pro-apoptotic protein. In the present study, we provide evidence that the E3 ubiquitin ligase Fbxo45 interacts with Par- 4 via its VASA-like sequence - a continuous six amino-acid peptide located in the N-terminal of Par-4 $\left({ }^{68} \mathrm{ELNNNL}^{72}\right)$, and that the interaction permits the polyubiquitylation and degradation of Par- 4 in cancer cells. Mutation of the Par-4 degron affects its interaction with and degradation by Fbxo45. Importantly, we demonstrate that the Par-4 degron mutant that is unable to bind Fbxo45 is stabilized and further enhances staurosporine-induced apoptosis of cancer cells.

Fbxo45 is the only F-box protein known to contain a SPRY domain. ${ }^{3}$ Besides Fbxo45, four mammalian suppressor of cytokine signaling (SOCS) box proteins (SSB-1 to -4) contain SPRY domains. The SOCS box-containing proteins, SSB-1 to SSB-4, belong to the SOCS box family of E3 ubiquitin ligases, which are likely to target proteins for ubiquitin-mediated degradation. Of these, SSB-1, SSB-2 and SSB-4, but not SSB-3 bind, with Par- $4,{ }^{30}$ and the interaction between SOCS box family proteins and Par-4 requires the ELNNN motif (VASA-like sequence) of Par-4. ${ }^{13,31}$ However, SSB-mediated regulation of Par-4 levels by proteasomal degradation has not been observed. ${ }^{30}$ In this study, we have demonstrated that Fbxo45 through its SPRY domain interacts with the VASA-like sequence of Par-4 and mediates it ubiquitylation and degradation by the proteasome.

The pro-apoptotic function of Par-4 requires phosphorylation of Par-4 at the T155 residue and its nuclear translocation. ${ }^{20}$ Cancer cells that exhibit impaired nuclear translocation of Par-4 are resistant to apoptosis. ${ }^{15}$ Nuclear translocation of Par-4 is essential for inhibition of NF-kB-dependent transcriptional activity; binding to Par-4 partner proteins WT1, ZIPK/ DAXX and THAP; and induction of apoptosis. ${ }^{15}$ AKT binding and phosphorylation of Par-4 result in its cytoplasmic retention and abrogation of apoptosis. ${ }^{21}$ Our studies show that Fbxo45 interacts with Par-4 in the cytoplasm (Figures $2 \mathrm{~b}$ and c), and mediates the proteasomal degradation of Par-4, which is phosphorylated by AKT. Accordingly, inhibition of AKT leads to nuclear translocation of Par- $4^{21}$ and attendant Par-4 accumulation in the nucleus (Figures $3 d$ and e) with consequent abrogation of Fbxo45 interaction (Figure 2c).

Overexpression of Par-4 induces apoptosis of cancer cells. ${ }^{15,16,21}$ Analysis of several mutants resulting from serial deletion of the full-length Par-4 protein from both the $\mathrm{N}$ - and C-termini led to the identification of a unique core domain (spanning amino acids 137-195), which was previously shown to be necessary and sufficient to induce apoptosis specifically in cancer cells. ${ }^{16,19}$ These studies identified a unique death-inducing domain selective for apoptosis induction (SAC domain), which holds promise for exploiting key differences between cancer and normal cells that could be employed for molecular therapy of cancer. ${ }^{16}$ A previous study demonstrated that Par-4 is cleaved by caspase-3 at D131, 
and the cleaved fragment of Par-4, but the not full-length Par-4, was found to accumulate in nuclear extracts after apoptosis induction. ${ }^{32}$ Based on our data, the cleaved fragment lacks the ELNNN degron motif and thus would be protected from Fbxo45-mediated degradation. Our studies further establish that cytoplasmic Par-4 undergoes Fbxo45-mediated degradation and that Par-4 translocation into nuclear compartment protects it from Fbxo45-mediated degradation. Notably, it has been demonstrated that cells expressing wild-type Par-4 undergo apoptosis but cells expressing the C-terminal fragment of Par-4 (aa131-aa333) show up to $20 \%$ enhancement of apoptosis. $^{32}$ Our studies suggested that the C-terminal fragment may potentiate a pro-apoptotic function because the absence of VASA-like sequence protects Par-4 from Fbxo45mediated proteasomal degradation.

Previous studies have demonstrated that Par-4 overexpression induces apoptosis and tumor regression. ${ }^{17,33}$ To assess the functional significance of Par-4 regulation by Fbxo45 in cancer cells, we established Tet-on- and Tet-offregulated expression of the Par-4 $3 \mathrm{~A}$ mutant, which was resistant to degradation by Fbxo45 in HeLa cells. Accumulation of Par-4 in HeLa cells caused significant apoptosis (Figures $5 \mathrm{a}$ and $\mathrm{b}$ ). Consistent with this result, co-expression of Fbxo45 with Par-4 attenuated Par-4-induced apoptosis (Figure 5c). These findings indicate that Fbxo45 suppresses cancer cell apoptosis through the degradation of Par-4. Using proliferation and colony formation assays, we also demonstrate that Fbxo45 attenuates the tumor-suppressor properties of Par-4 (Figure 6).

In conclusion, we report that the E3 ligase Fbxo45 interacts with Par-4 through its VASA-like sequence in the cytoplasm and mediates its ubiquitylation and proteasomal degradation. Our findings uncover a hitherto unknown mechanism for the regulation of apoptosis in cancer cells via proteasomal degradation of Par-4. These results carry implication for potential exploitation of the Fbxo45-Par-4 axis in human cancer treatment.

\section{Materials and Methods}

Plasmids. Fbxo45 cDNA was cloned from a K562 cell line into the pENTR/DTOPO vector, and subcloned into a Streptavidin-Biotin-HA tandem-affinity purification (TAP)-tagged vector (kindly provided by Dr. Stephane Angers). Fulllength $\beta$-TrCP and Skp2 constructs were kindly provided by Dr. Michele Pagano and were cloned in the pIRESpuro-GLUE vector. FLAG-tagged Fbxw4, FLAGtagged Fbxw5 and FLAG-tagged Fbxw7 were kindly provided by Dr. Michele Pagano. Skp1 and Par-4 clones were purchased from Open Biosystems (Pittsburgh, PA, USA). Full-length Par-4, deletion mutants and point mutants of Par-4 were cloned in pcDNA3.1-nV5 (Invitrogen, Grand Island, NY, USA) for biochemistry experiments, and also cloned in Lenti-X Tet-On Advanced Inducible Expression System (Clontech, Mountain View, CA, USA) for biological experiments. Point mutants were generated using GeneTailor Site-Directed Mutagenesis System (Invitrogen). The deletion mutants were prepared by standard PCR procedures.

Immunoprecipitation and immunoblotting. For immunoprecipitation, cells were lysed for $20 \mathrm{~min}$ in IP buffer containing $50 \mathrm{mM}$ HEPES pH 8.0, $150 \mathrm{mM}$ $\mathrm{NaCl}, 2 \mathrm{mM}$ EDTA, 1\% Nonidet P40, $2 \mathrm{mM}$ DTT and protease inhibitor cocktail (Calbiochem, Billerica, MA, USA) on ice to get the whole-cell lysate. The cytoplasmic lysate or the nuclear lysate was extracted using NE-PER Nuclear and Cytoplasmic Extraction Reagents (Pierce, Rockford, IL, USA). Cell lysates were incubated with the indicated antibodies for $2 \mathrm{~h}$ to overnight at $4{ }^{\circ} \mathrm{C}$ and protein complexes were immunoprecipitated by Protein A/G agarose beads (Santa Cruz, Dallas, TX, USA). Immunoprecipitates were washed five times with IP buffer, eluted by heat denaturation in SDS sample buffer and analyzed by immunoblotting. After immunoblotting, blocking and antibody incubation were carried out in $5 \%$ non-fat milk in PBST, and washings in PBST. Blots were developed using ECL buffer (Thermo Scientific, Rockford, IL, USA) following the instructions of the manufacturer.

Antibody. The following antibodies were used: anti-HA (Covance, Daytona Beach, FL, USA), anti-FLAG (Sigma, St. Louis, MO, USA), anti-V5 (Invitrogen), anti-Actin (Sigma), anti-Par-4 (Abcam, Cambridge, MA, USA), anti-Skp1 (BD, San Jose, CA, USA), anti-PARP-1 B (Sigma), anti-AKT (Cell Signalling, Danvers, MA, USA), anti-P-AKT (Cell Signalling). Fbxo45 antibody was generated by Thermo Scientific Company. The peptide sequence was used to generate antisera as previously described. ${ }^{10}$

Flow cytometry analysis. For measuring apoptosis, transfected cells were dual stained with 7-AAD (PE) and APC-Annexin V using an Alexa Fluor 488annexin V Dead Cell apoptosis kit (Invitrogen) according to the manufacturer's protocol. Stained cells were analyzed by flow cytometry (FC 500 MPL system; Beckman Coulter, Brea, CA, USA).

In situ TUNEL assay. Apoptosis was determined by detecting DNA fragmentation using the TUNEL Assay System (Roche, Indianapolis, IN, USA) in accordance with the manufacturer's instructions. Briefly, cultured cells grown on coverslips were fixed with paraformaldehyde (4\% in PBS) for $15 \mathrm{~min}$ and permeabilized with PBT $(0.1 \%$ Triton X-100 in PBS) for $10 \mathrm{~min}$. The cells were then incubated in TUNEL reaction mixture for $60 \mathrm{~min}$ at $37^{\circ} \mathrm{C}$. After three washes with PBS, samples were directly analyzed under the microscope.

Interactome identification using mass spectrometry. Immunoprecipitated samples (details) were resolved on SDS-PAGE, and visualized by Coomasssie stain. In-gel digestion by trypsin was carried out using procedure standardized at the Proteomics Resource Facility (University of Michigan). Tryptic peptides were resolved on a nano-capillary reverse phase column and introduced directly into a linear ion-trap mass spectrometer (LTQ XL, Thermo Fisher, Waltham, MA, USA). The mass spectrometer was set to collect one survey scan (MS1), followed by MS/MS spectra on the nine most intense ions observed in MS1 scan. Proteins were identified by searching the MS/MS spectra against human protein database using X!Tandem/TransProteomic Pipeline software suite developed by ISB (Seattle, WA, USA). All proteins identified with a probability of $\geq 0.9$ were kept for further analysis.

Cell culture and drug treatment. 293T, HeLa and PC3 cells were grown in DMEM (Invitrogen), supplemented with 10\% fetal bovine serum (Invitrogen) and $0.2 \mathrm{mM}$ L-glutamine (Invitrogen). Fbxo45 gene trap mES cells (SIGTR ES cell line AZ0252) were obtained from Mutant Mouse Regional Resource Center (MMRRC) program. The mES cells were grown in $1 \times$ GMEM medium (Sigma) supplemented with $2 \mathrm{mM}$ L-glutamine (Invitrogen), $1 \mathrm{mM}$ sodium pyruvate (Invitrogen), $1 \times$ nonessential amino acids (Invitrogen), 10\% (v/v) fetal bovine serum (Invitrogen), a 1:1000 dilution of $\beta$-mercaptoethanol stock solution, and $500-1000 \mathrm{U} / \mathrm{ml}$ of leukocyte inhibitory factor (Chemicon, Temecula, CA, USA). The following drugs were used: proteasome inhibitor MG132 (Sigma), CAL-101 (AKT inhibitor; Selleckchem, Houston, TX, USA), staurosporine (Sigma), doxycycline (Sigma) and cycloheximide (Sigma).

Transient transfection and lentivirus-mediated gene transfer. Cells were transfected with the use of Lipofectamine reagent (Invitrogen) or Poly jet (Signagen, Rockville, MD, USA). For lentivirus-mediated gene transfer, 293T cells were co-transfected with lentiviral vector together with packaging vectors. At $48 \mathrm{~h}$ after transfection, virus-containing medium was collected and supplemented with Polybrene $(4 \mu \mathrm{g} / \mathrm{ml}$; Sigma). Cells were then infected with the viral supernatant. The transiently transfected cells and/or the lentivirus-mediated transduced cells were selected with Puromycin $(2 \mu \mathrm{g} / \mathrm{ml})$ for $7-14$ days to generate the stable gene-expressing cell lines.

Gene silencing by shRNA or siRNA. Lentiviral shRNA against Fbxo45 were purchased from Open Biosystems (V2LHS_182000, V2LHS_222040, V2LHS_254545).

GenomeWide siRNA of Fbxo45 was 140186437_10, Human Fbxo45 (NM_00115573.1) sequence of sense strand

5'-CAGAUAGGAGAAAGAAUUCGA-3'. 
As a control for potential off-target effects, a pool of four siCONTROL non-targeting siRNAs was used (D-001206-13).

5'-AUGAACGUGAAUUGCUCAA-3'
5'-UAAGGCUAUGAAGAGAUAC-3'
5'-AUGUAUUGGCCUGUAUUAG-3'

5'-UAGCGACUAAACACAUCAA-3'.

Real-time PCR. RNA was isolated from cells using RNeasy Mini kits (Qiagen, Valencia, CA, USA). cDNA was synthesized using SuperScript kit (Invitrogen). The following primer pairs were used for real-time PCR amplification:

Human Fbxo45:

5'-AGTGCCAAGGTTATGTGGCATTGCTG-3'

5'-AGAAAGCCACTGTCATCCGTCCAAAG-3'

Quantitative real-time PCR was carried out using SYBR green (Invitrogen). Reactions were performed in triplicate according to the manufacturer's protocol. GAPDH was used as the internal control.

In vivo ubiquitylation assay. $293 \mathrm{~T}$ cells were transfected with expression vectors encoding Skp1, HA-tagged Fbx045, FLAG-tagged ubiquitin and V5-tagged wild-type Par-4 or mutant Par-4 (3A). At $48 \mathrm{~h}$ after transfection, cells were incubated with MG132 for $4 \mathrm{~h}$ before lysis. WCEs were subjected to immunoprecipitation with anti-V5 resin followed by blotting with the indicated antibodies.

The in vitro ubiquitylation assay. The in vitro ubiquitylation assay was performed with In vitro-translated Flag-Par-4 and its corresponding mutants as substrate. For ligase purification, TAP HA-tagged Fbxo45 E3 ligase complex was expressed and immunoprecipitated from 293T cells. Immunoprecipitates were further used for in vitro ubiquitylation assays in a volume of $100 \mu \mathrm{l}$, containing $2 \mathrm{mM}$ ATP, $1.5 \mathrm{mg} / \mathrm{ml} \mathrm{E1}, 10 \mathrm{mg} / \mathrm{ml}$ E2 (Ubch5c) and (Ube2w), $1 \mu \mathrm{M}$ ubiquitin aldehyde, $2.5 \mu \mathrm{g} / \mu$ Flag-tagged ubiquitin and protease inhibitor cocktail. The reactions were incubated at $30^{\circ} \mathrm{C}$ for $45 \mathrm{~min}$ and analyzed by immunoblotting.

Normalization and quantification of protein levels. Protein concentrations of WCE were performed using a BCA protein assay kit (Thermo Scientific). Protein bands were scanned and quantified using Imagin J software. All data represent the average from at least three independent experiments.

Statistical analyses. All data represent the average from at least three independent experiments. Significance was calculated by using the double-tailed $t$-test. Differences were considered significant when $P$ was $<0.05$.

Soft-agar colony assay. Cells were re-suspended in DMEM containing $10 \%$ fetal bovine serum along with $0.35 \%$ low-melting agarose and seeded on a plate coated with $1 \%$ agarose in DMEM and $10 \%$ fetal bovine serum. Viable colonies were scored after 3 weeks of incubation and the quantified data were presented from three independent experiments.

Cell proliferation assay. Cells were seeded in 96-well plates. Plating medium to provide 5000 plated cells/well in a final volume of $100 \mu$ l. After $24 \mathrm{~h}$ of seeding, cellular ATP concentrations were assessed using the Cell Titer-Glo Luminescent Cell Viability Assay (Promega, Madison, WI, USA). Luminescence readings were taken on the Tecan GENios Pro microplate reader (Tecan, San Jose, CA, USA).

\section{Conflict of Interest}

The authors declare no conflict of interest.

Acknowledgements. We thank Dr. Joon-Young Ahn for his assistance with the characterization of the anti-Fbxo45 polyclonal antibody. This work was funded by NIH grants R01 DE119249 and R01 CA136905 to KSJE-J and R01 CA140806 to MSL

1. Bai C, Sen P, Hofmann K, Ma L, Goebl M, Harper JW et al. SKP1 connects cell cycle regulators to the ubiquitin proteolysis machinery through a novel motif, the F-box. Cell 1996; 86: 263-274.

2. Cenciarelli C, Chiaur DS, Guardavaccaro D, Parks W, Vidal M, Pagano M. Identification of a family of human F-box proteins. Curr Biol 1999; 9: 1177-1179.
3. Jin J, Cardozo T, Lovering RC, Elledge SJ, Pagano M, Harper JW. Systematic analysis and nomenclature of mammalian F-box proteins. Genes Dev 2004; 18 : 2573-2580.

4. Skowyra D, Craig KL, Tyers M, Elledge SJ, Harper JW. F-box proteins are receptors that recruit phosphorylated substrates to the SCF ubiquitin-ligase complex. Cell 1997; 91 : 209-219.

5. Vashisht AA, Zumbrennen KB, Huang X, Powers DN, Durazo A, Sun D et al. Control of iron homeostasis by an iron-regulated ubiquitin ligase. Science 2009; 326 : 718-721.

6. Wertz IE, Kusam S, Lam C, Okamoto T, Sandoval W, Anderson DJ et al. Sensitivity to antitubulin chemotherapeutics is regulated by MCL1 and FBW7. Nature 2011; 471: $110-114$.

7. Liao EH, Hung W, Abrams B, Zhen M. An SCF-like ubiquitin ligase complex that controls presynaptic differentiation. Nature 2004; 430: 345-350.

8. Saiga T, Fukuda T, Matsumoto M, Tada H, Okano HJ, Okano H et al. Fbxo45 forms a novel ubiquitin ligase complex and is required for neuronal development. Mol Cell Biol 2009; 29: 3529-3543.

9. Peschiaroli A, Scialpi F, Bernassola F, Pagano M, Melino G. The F-box protein FBXO45 promotes the proteasome-dependent degradation of p73. Oncogene 2009; 28 : 3157-3166.

10. Tada H, Okano HJ, Takagi H, Shibata S, Yao I, Matsumoto $M$ et al. Fbxo45, a novel ubiquitin ligase, regulates synaptic activity. J Biol Chem 2010; 285: 3840-3849.

11. Ponting $\mathrm{C}$, Schultz J, Bork P. SPRY domains in ryanodine receptors $(\mathrm{Ca}(2+)$-release channels). Trends Biochem Sci 1997; 22: 193-194.

12. Rhodes DA, de Bono B, Trowsdale J. Relationship between SPRY and B30.2 protein domains. Evolution of a component of immune defence? Immunology 2005; 116: $411-417$.

13. Woo JS, Suh HY, Park SY, Oh BH. Structural basis for protein recognition by B30.2/SPRY domains. Mol Cell 2006; 24: 967-976.

14. Sells SF, Wood DP Jr., Joshi-Barve SS, Muthukumar S, Jacob RJ, Crist SA et al. Commonality of the gene programs induced by effectors of apoptosis in androgen-dependent and -independent prostate cells. Cell Growth Differ 1994; 5: 457-466.

15. El-Guendy N, Rangnekar VM. Apoptosis by Par-4 in cancer and neurodegenerative diseases. Exp Cell Res 2003; 283: 51-66.

16. El-Guendy N, Zhao Y, Gurumurthy S, Burikhanov R, Rangnekar VM. Identification of a unique core domain of par-4 sufficient for selective apoptosis induction in cancer cells. $\mathrm{Mol}$ Cell Biol 2003; 23: 5516-5525.

17. Chakraborty M, Qiu SG, Vasudevan KM, Rangnekar VM. Par-4 drives trafficking and activation of Fas and Fasl to induce prostate cancer cell apoptosis and tumor regression. Cancer Res 2001; 61: 7255-7263.

18. Nalca A, Qu SG, El-Guendy N, Krishnan S, Rangnekar VM. Oncogenic Ras sensitizes cells to apoptosis by Par-4. J Biol Chem 1999; 274: 29976-29983.

19. Diaz-Meco MT, Lallena MJ, Monjas A, Frutos S, Moscat J. Inactivation of the inhibitory kappaB protein kinase/nuclear factor kappaB pathway by Par-4 expression potentiates tumor necrosis factor alpha-induced apoptosis. J Biol Chem 1999; 274: 19606-19612.

20. Gurumurthy S, Goswami A, Vasudevan KM, Rangnekar VM. Phosphorylation of Par-4 by protein kinase A is critical for apoptosis. Mol Cell Biol 2005; 25: 1146-1161.

21. Goswami A, Burikhanov R, de Thonel A, Fujita N, Goswami M, Zhao Y et al. Binding and phosphorylation of par-4 by akt is essential for cancer cell survival. Mol Cell 2005; 20: 33-44.

22. Ranganathan $P$, Rangnekar VM. Regulation of cancer cell survival by Par-4. Ann N Y Acad Sci 2005; 1059: 76-85.

23. Moreno-Bueno G, Fernandez-Marcos PJ, Collado M, Tendero MJ, Rodriguez-Pinilla SM, Garcia-Cao I et al. Inactivation of the candidate tumor suppressor par-4 in endometrial cancer. Cancer Res 2007; 67: 1927-1934.

24. Garcia-Cao I, Lafuente MJ, Criado LM, Diaz-Meco MT, Serrano M, Moscat J. Genetic inactivation of Par4 results in hyperactivation of NF-kappaB and impairment of JNK and p38. EMBO Rep 2003; 4: 307-312.

25. Zhao Y, Burikhanov R, Qiu S, Lele SM, Jennings CD, Bondada $S$ et al. Cancer resistance in transgenic mice expressing the SAC module of Par-4. Cancer Res 2007; 67: 9276-9285.

26. Alvarez JV, Pan TC, Ruth J, Feng Y, Zhou A, Pant D et al. Par-4 downregulation promotes breast cancer recurrence by preventing multinucleation following targeted therapy. Cancer Cell 2013; 24: 30-44.

27. Wu C, Daniels RW, DiAntonio A. DFsn collaborates with Highwire to down-regulate the Wallenda/DLK kinase and restrain synaptic terminal growth. Neural Dev 2007; 2: 16.

28. Styhler S, Nakamura A, Lasko P. VASA localization requires the SPRY-domain and SOCS-box containing protein, GUSTAVUS. Dev Cell 2002; 3: 865-876.

29. Craig AG, Bandyopadhyay P, Olivera BM. Post-translationally modified neuropeptides from Conus venoms. Eur J Biochem 1999; 264: 271-275.

30. Masters SL, Yao S, Willson TA, Zhang JG, Palmer KR, Smith BJ et al. The SPRY domain of SSB-2 adopts a novel fold that presents conserved Par-4-binding residues. Nat Struct Mol Biol 2006; 13: 77-84. 
31. Filippakopoulos P, Low A, Sharpe TD, Uppenberg J, Yao S, Kuang Z et al. Structural basis for Par-4 recognition by the SPRY domain- and SOCS box-containing proteins SPSB1, SPSB2, and SPSB4. J Mol Biol 2010; 401: 389-402.

32. Chaudhry P, Singh M, Parent S, Asselin E. Prostate apoptosis response 4 (Par-4), a novel substrate of caspase-3 during apoptosis activation. Mol Cell Biol 2012; 32: 826-839.
33. Azmi AS, Wang Z, Burikhanov R, Rangnekar VM, Wang G, Chen J et al. Critical role of prostate apoptosis response-4 in determining the sensitivity of pancreatic cancer cells to small-molecule inhibitor-induced apoptosis. Mol Cancer Ther 2008; 7: 2884-2893.

34. Sahasrabuddhe AA, Dimri M, Bommi PV, Dimri GP. betaTrCP regulates BMl1 protein turnover via ubiquitination and degradation. Cell Cycle 2011; 10: 1322-1330.

Supplementary Information accompanies this paper on Cell Death and Differentiation website (http://www.nature.com/cdd) 